\title{
Growth of InAs Wurtzite Nanocrosses from Hexagonal and Cubic Basis
}

Krizek, Filip; Kanne, Thomas; Razmadze, Davydas; Johnson, Erik; Nygaard, Jesper; Marcus, Charles M; Krogstrup, Peter

\section{Published in:}

Nano Letters

Link to article, DOI:

10.1021/acs.nanolett.7b02604

Publication date:

2017

Document Version

Peer reviewed version

Link back to DTU Orbit

Citation (APA):

Krizek, F., Kanne, T., Razmadze, D., Johnson, E., Nygaard, J., Marcus, C. M., \& Krogstrup, P. (2017). Growth of InAs Wurtzite Nanocrosses from Hexagonal and Cubic Basis. Nano Letters, 17(10), 6090-6096.

https://doi.org/10.1021/acs.nanolett.7b02604

\section{General rights}

Copyright and moral rights for the publications made accessible in the public portal are retained by the authors and/or other copyright owners and it is a condition of accessing publications that users recognise and abide by the legal requirements associated with these rights.

- Users may download and print one copy of any publication from the public portal for the purpose of private study or research.

- You may not further distribute the material or use it for any profit-making activity or commercial gain

- You may freely distribute the URL identifying the publication in the public portal

If you believe that this document breaches copyright please contact us providing details, and we will remove access to the work immediately and investigate your claim 


\section{Communication}

Subscriber access provided by DTU Library

\section{Growth of InAs Wurtzite Nanocrosses from Hexagonal and Cubic Basis}

Filip Krizek, Thomas Kanne, Davydas Razmadze, Erik Johnson, Jesper Nygård, Charles M. Marcus, and Peter Krogstrup

Nano Lett., Just Accepted Manuscript • DOI: 10.1021/acs.nanolett.7b02604 • Publication Date (Web): 12 Sep 2017

Downloaded from http://pubs.acs.org on September 17, 2017

\section{Just Accepted}

"Just Accepted" manuscripts have been peer-reviewed and accepted for publication. They are posted online prior to technical editing, formatting for publication and author proofing. The American Chemical Society provides "Just Accepted" as a free service to the research community to expedite the dissemination of scientific material as soon as possible after acceptance. "Just Accepted" manuscripts appear in full in PDF format accompanied by an HTML abstract. "Just Accepted" manuscripts have been fully peer reviewed, but should not be considered the official version of record. They are accessible to all readers and citable by the Digital Object Identifier (DOI®). "Just Accepted" is an optional service offered to authors. Therefore, the "Just Accepted" Web site may not include all articles that will be published in the journal. After a manuscript is technically edited and formatted, it will be removed from the "Just Accepted" Web site and published as an ASAP article. Note that technical editing may introduce minor changes to the manuscript text and/or graphics which could affect content, and all legal disclaimers and ethical guidelines that apply to the journal pertain. ACS cannot be held responsible for errors or consequences arising from the use of information contained in these "Just Accepted" manuscripts. 


\title{
Growth of InAs Wurtzite Nanocrosses from
}

\section{Hexagonal and Cubic Basis}

\author{
Filip Krizek ${ }^{1}$, Thomas Kanne ${ }^{1}$, Davydas Razmadze ${ }^{1}$,Erik Johnson ${ }^{1,2}$, Jesper Nygård ${ }^{1}$, Charles M. \\ Marcus $^{1}$, Peter Krogstrup ${ }^{* 1}$ \\ ${ }^{1}$ Center for Quantum Devices and Station Q Copenhagen, Niels Bohr Institute, University of \\ Copenhagen, 2100 Copenhagen, Denmark \\ ${ }^{2}$ Department of Wind Energy, Technical University of Denmark, DTU Risø Campus, 4000 \\ Roskilde, Denmark
}

\begin{abstract}
Keywords: kinked nanowires, branched nanowires, nanowire junctions, nanowire shadow mask, nanowire networks
\end{abstract}

\begin{abstract}
Epitaxially connected nanowires allow for design of electron transport experiments and applications beyond the standard two terminal device geometries. In this letter, we present growth methods of three distinct types of wurtzite structured InAs nanocrosses via the Vapor-Liquid-Solid mechanism. Two methods use conventional wurtzite nanowire arrays as a six-fold hexagonal basis for growing single crystal wurtzite nanocrosses. A third method uses the two-fold cubic symmetry of (100) substrates to form well-defined coherent inclusions of zinc-blende in the center of the nanocrosses. We show that all three types of nanocrosses can be transferred undamaged to arbitrary substrates, which allows for structural, compositional and electrical characterization. We further demonstrate the
\end{abstract}




\section{potential for synthesis of as-grown nanowire networks and for using nanowires as shadow masks for in-situ fabricated junctions in radial nanowire heterostructures.}

Crystal growth of free-standing nanowires (NWs) offers a variety of flexible material designs, with a possibility to synthesise compositional and structural heterostructures, both axial and radial $^{1,2,3,4,5}$. On the other hand, controlled growth of NW based networks in geometries beyond two-terminal device designs is not as flexible as its planar counterpart, where the top-down approaches can be in principle used to define arbitrary networks ${ }^{6}$. However, there is a desire to combine the advantages of free-standing nanowires with the multi-terminal device geometries, which has inspired several research groups to focus on in-situ growth of branched and epitaxially interconnected III-V NWs $7,8,9,10,11,12,13,14,15,16,17$. Progressing towards advanced free-standing nanostructures, such as branched T-shaped NWs and X-shaped nanocrosses (NCs), requires new approaches to crystal growth and substrate preparation. Moreover, the crystal structure and morphology of the junctions becomes important, as it affects the electrical properties of the structures and therefore their potential in relevant applications, e.g. coherent quantum transport experiments $^{18}$. In general, a single crystal NC can be formed if the crystal structure of the merging NWs falls within a subgroup of the substrate symmetry group. The majority of the current development relies on growth along the two-fold [111]B directions on cubic (100) substrates, where the single crystallinity can be achieved, if the NWs grow in the ZB structure from a ZB substrate and therefore preserve the symmetry. In contrast, if the NWs grow in the hexagonal WZ structure from the cubic basis of a ZB substrate, the two sets of NWs will have different crystal orientations. That will lead to an incoherent match between the merging NWs, which can drive a crystal reconstruction back to the cubic symmetry ${ }^{13,16,19}$. 
In this letter, we present a strategy for growing interconnected single crystal InAs NW NCs in the WZ structure. The growth is achieved by using [0001]B NWs as a platform for kinking InAs WZ NWs in parallel to the substrate ${ }^{5,20,21,22}$ (Figure 1a). The crystal structure of the kinked NWs, which in these experiments preferentially grow along one of the six $<1-100>$ directions, is then locked into the WZ parent basis. Relative positioning of the seed particles with respect to the substrate crystal axes that correspond to the six-fold growth directionality, enables growth of X-shaped 'kinked-planar' ([1-100]X[01-10]) (Figure 1b) and 'kinked-vertical' ([1100]X[0001]) (Figure 1d) NW NCs, which are presented in the first part of the letter. In the second part we present growth of hetero-crystal WZ/ZB/WZ ([0001]X[0001]) NCs (Figure 3a), which originates from [0001]B WZ NWs grown directly from the two-fold [111]B directions on (100) substrates. Finally, we discuss the possibilities to grow complex NW networks and to form controlled NW shadow masks for junctions in core-shell NWs.

The NWs were grown on either bare or oxide masked InAs (111)B and InP (100) substrates by molecular beam epitaxy (MBE) via vapor-liquid-solid (VLS) method in a GEN II Varian system. The substrates were pre-patterned with $\mathrm{Au}$ particles using electron beam lithography (EBL). More details of substrate fabrication are given in the supplementary information (S1). The prepatterned substrates were plasma ashed in oxygen and cleaned in 20:1 $\mathrm{H}_{2} \mathrm{O}: \mathrm{HF}$ solution prior to growth. The native oxide was desorbed by annealing under As overpressure at $540^{\circ} \mathrm{C}$. The [0001] NWs on masked InP (100) substrates were grown at substrate temperature of $410^{\circ} \mathrm{C}$, an In flux corresponding to a planar growth rate of $0.7 \mu \mathrm{m} / \mathrm{hr}$ and a $\mathrm{As}_{2} / \mathrm{In}$ ratio of 20 . NWs on InAs (111)B substrates were grown at $420^{\circ} \mathrm{C}$ and $\mathrm{As}_{4} / \mathrm{In}$ ratio of 30 . The growth direction can be changed in various ways, as shown in previous works ${ }^{14,23,24,25,26,27,28,29,30,31,32}$, where adjustment 
of either temperature, fluxes or liquid composition can result in an abrupt change in the liquid wetting (see ref. $[33,34]$ for discussion on wetting mechanisms). In this work we change the NW growth direction by introducing a short growth period of GaAs. ${ }^{22}$ The probability of changing the NW growth into the $<1-100>$ direction, or maintaining [0001]B direction, depends on the amount of introduced Ga. The growth of the NWs then continues along the kinked $<1-100>$ or the vertical $<0001>$ directions after reintroduction of the In flux. We gradually decrease both the In and As fluxes during the kinked NW growth, to reduce NW tapering as discussed below.
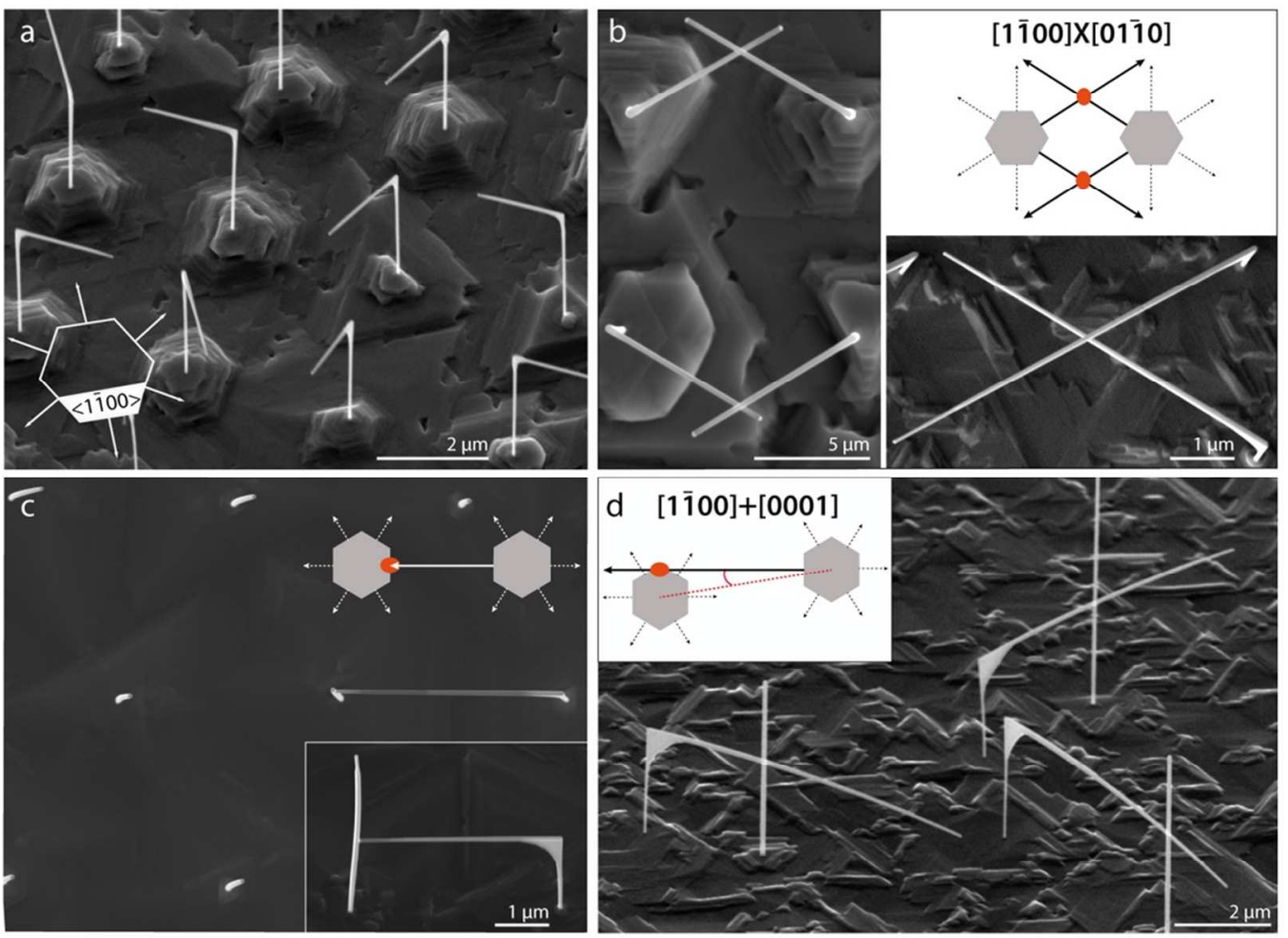

Figure 1. Growth of NCs in WZ basis a) Tilted SEM image of a kinked InAs NW array grown on a InAs (111)B substrate. b) Top-view SEM images of 'kinked-planar' InAs NCs ([1100]X[01-10]) formed from two kinked NWs crossing in a plane parallel to the substrate. c) Topview SEM image of a hexagonally patterned NW array, grown with a small probability to kink, which yields a vertical InAs 'h-structure' (formed from kinked and free-standing NWs - [1$100] \mathrm{T}[0001]$ ), as seen in the tilted side-view inset. d) A slight rotation of the hexagonal seed 
pattern with respect to the [11-2] crystal direction promotes formation of 'kinked-vertical' NCs $([1-100]+[0001])$.

For the synthesis of kinked-planar junctions the growth direction is kinked into one of the six $<1-100>$ directions parallel to the growth substrate (Figure 1a). In this work, we get a NW kinking probability up to $100 \%$ by introducing a Ga flux for 15 s, corresponding to a GaAs planar growth of $2.25 \mathrm{~nm}$. Note that the six growth directions form with equal probability and are defined by the faceting of the [0001]B NWs. High yield of the NCs based on kinked NWs is obtained by positioning the seed particles along the six $<11-2>$ crystal directions of the substrate, as illustrated in Figure 1 (b, d and a specific example of a hexagonal array in c). This allows for the kinked NWs grow and merge in the same WZ basis. Thus, when the probability to kink is high, two NWs kinked at the same height parallel to the substrate can merge into an X shaped kinked-planar NC (Figure 1b). The ratio of kinked and straight NWs can be tuned by varying the GaAs growth time. When the fraction of the kinked NWs is significantly reduced, vertical hshaped structures can be grown from a hexagonal pattern, as shown in Figure 1c for 4 second growth of GaAs. In this scheme the kinked NW directly hits one of the $\{1-100\}$ side facets of the [0001]B NW that maintained the initial growth direction and forms a T shaped junction ([1$100] \mathrm{T}[0001])$. If the same principle is used in combination with a slight rotation of the hexagonal seed pattern in the EBL design, the kinked NW passes the [0001]B NW while growing in a distance set by the rotation (50 to $300 \mathrm{~nm}$ ). Both NWs subsequently bond together and form + shaped kinked-vertical NC (Figure 1d). The kinked-planar junctions are similarly formed from two NWs passing close one to another in different height. Therefore, the seed nanoparticles play no role in the merging process of the NWs and none of the NC junctions are 
contaminated by the seed material. A similar form of in-situ NW attraction was previously reported by Dai et. al. ${ }^{35}$ We stress, that precise substrate patterning combined with control of the kinking probability greatly increases the yield of both types of structures.

The single crystalline junctions of the kinked-vertical and kinked-planar NCs are modelled in Figure 2 (a) and (e), respectively. In supplementary information S2, we show that it is possible to transfer both types of the NCs by micro-mechanical manipulator needles to various substrates with high precision, e.g. to TEM grids as shown in Figure 2 (b, f). Figure $2 \mathrm{c}$ shows a TEM image of kinked-vertical junction taken along the [0-110] axis projection. As shown in supplementary information S3, the NWs are grown together via the facets and not the bulk, which is also reflected by the darker contrast in the junction due to the increased thickness caused by the overlapping NWs. However, these NCs appears just as mechanical stable as fully overgrown NCs, judging solely from the mechanical transfer. In Figure 2c, the NWs forming this particular examined junction are rotated with respect to each other (visible from Moiré patterns and diffraction patterns of the junction in Figure 2d). It is likely that when the kinked $\mathrm{NC}$ junctions are affected by inter-wire rotation, although the crystal coherency can be retained by strain, which is supported by the fact that we did not observe any signs of dislocations. Apart from the inter-wire rotations, the structural coherency of the junction will be affected by the presence of different polytypes and stacking faults. One of the typical issues we observe is radial growth of ZB structure on the (0001)B top-facet. The onset of the radial growth can be related to the critical adatom density on the NW sidefacets, which depends exponentially on both group III and group V fluxes, as described in ref. [34]. The tapering thus initiates when the kinked NW grows longer than a given effective adatom diffusion length, corresponding to the critical adatom 
density. The radial growth tends to start at the corner of the kink and propagate outwards (see supplementary information S4). The stacking faults in the kinked NWs are inherited from the [0001]B NW stem and propagate in parallel to the [0001]B plane. Even though Figure 2(h) shows a perfect match between the two kinked nanowires, diffraction analysis and high resolution TEM characterization of the kinked-planar junction (Figure 2g) along the [0001] zone axis projection (Figure 2h), shows A-B-C stacking sites, indicating presence of ZB stackingfaults in the structure. In order to obtain single crystal non-tapered NCs, growth conditions that yield stacking fault free [0001]B NWs and at the same time longer adatom diffusion lengths during kinked NW growth, are of great importance. Interestingly, we have not observed interwire rotations in the kinked-planar junctions.

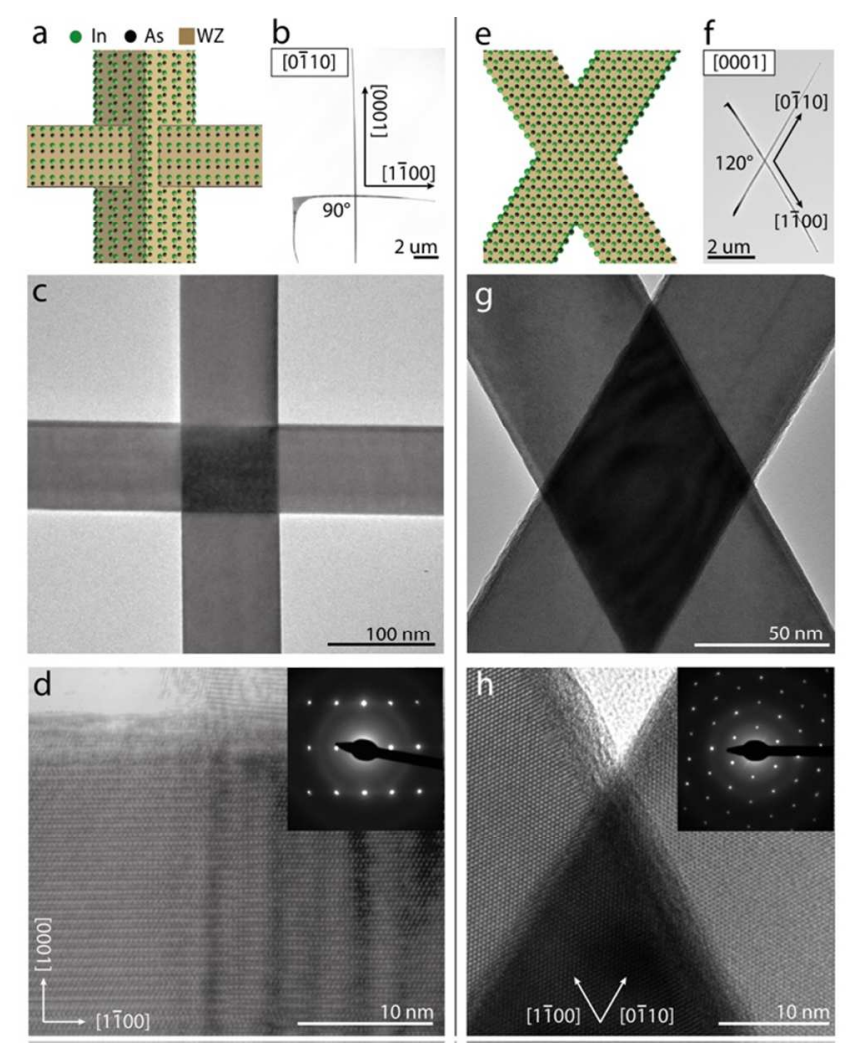

Figure 2. TEM of NCs grown in hexagonal basis. a) WZ crystal structure model of a kinkedvertical NC, shown on TEM grid in b). c) Zoom-in on the junction region of the same NC with visible Moiré pattern and further high resolution zoom-in onto the junction corner d), showing two slightly rotated WZ crystals. The inset displays a diffraction pattern of the junction. e) WZ 
crystal structure model of a kinked-planar NC, shown on TEM grid in f). g) Zoom-in on the junction region of the kinked-planar $\mathrm{NC}$ and further zoom-in on one of the junction corners $\mathrm{h}$ ) showing single crystalline WZ nature. The inset shows a diffraction pattern of the junction.

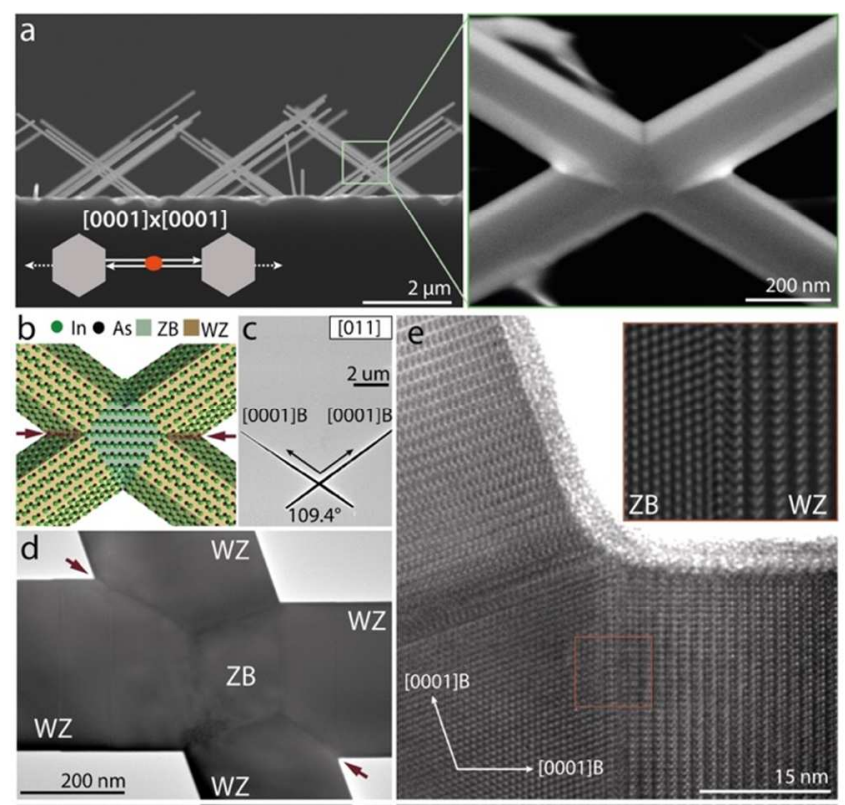

Figure 3. InAs WZ [0001]B NCs grown from a cubic basis. a) Cross-sectional SEM image of InAs 'WZ/ZB/WZ' NCs ([0001]X[0001]) grown from seed particles deposited along the [0-10] direction on InP (100) substrate (left panel). A close up SEM image of the junction (right panel). b) Crystal structure model of the WZ/ZB/WZ junction showing coherent WZ-ZB-WZ transition and incoherent WZ-WZ boundaries (red arrows). c) A low resolution TEM overview of the NC shown in d) with a visible ZB segment in the center as shown in the high resolution TEM image in e) depicting the WZ-ZB-WZ transition in the corner of the junction. The inset in e) also shows the well-defined single plane transition from WZ to ZB.

(100) substrates have been previously used to grow a variety of nanostructures, e.g. vertical $\mathrm{NWs}^{36,37}$, inclined $\mathrm{NWs}^{13,36}$ or nano-plates ${ }^{38}$. In this study, we use parameters that promote the InAs nanowire growth along the two $<111>\mathrm{B}$ directions on InP substrates and subsequent formation of $\mathrm{WZ} / \mathrm{ZB} / \mathrm{WZ}$ NCs. We find that a relatively high $\mathrm{V} / \mathrm{III}$ ratio combined with an oxide masked substrate ${ }^{39,40}$ increases the yield of these inclined NWs. However, the initial growth depends on the details of the catalyst interaction with the substrate ${ }^{41,42}$, which also 
influence the preferential NW morphology and crystal structure. We also find that the NW crystal structure changes from $\mathrm{ZB}$ to pure WZ during the initial growth stage, under the given growth conditions ${ }^{13,43,44}$. Growth of two NWs along the opposite $<111>\mathrm{B}$ directions, stemming from seed particles positioned along the [0-11] direction, then results in formation of the vertical X-shaped WZ/ZB/WZ NCs (Figure 3a left panel). A small NW diameter determined by the size of the seed particles, enables them to pass one another in close proximity, bond together and overgrow into a cross during further growth. Because the relevant symmetry point sub-group of WZ has a lower symmetry than that of ZB, the two merging WZ NWs, originating from the two [111]B orientations of a cubic substrate, grow in two differently rotated crystal bases. This leads to a crystal incoherency which acts as a driving force for recrystallization into the cubic symmetry $^{13,16,19}$. The recrystallized junction consists of a highly reproducible 3D diamond shaped ZB inclusion surrounded by WZ/WZ incoherent boundaries, as illustrated in the crystal model in Figure 3b. This can occur without further disruption of the WZ [0001]B NW growth and a faceted homogeneous junction is formed (Figure 3a right panel). The crystal model together with TEM of the junction (Figure 3d), clearly shows two incoherent WZ-WZ boundaries left behind after recrystallization and surrounding the ZB diamond. These incoherent grain boundaries persist in the structure, because further recrystallization is either kinetically limited or just energetically unfavourable. The WZ-ZB-WZ transition makes a coherent single atomic layer interface as shown in Figure 3e and in detail in supplementary information S5.

While the NCs grown on (100) substrates (Figure 5a) are suitable for merging multiple nanowires ${ }^{17}$, the kinked NW growth additionally has potential for upscaling into larger as-grown NW networks, because of the planar geometry. This requires control of the height of the kink 
(Figure 4a), which depends on the uniformity of the NW stems. The growth rate depends on the NW diameter which is related to the volume of the seed particles. Thus, the height of the kinks is controlled by the amount of Au deposited in the holes defined in the EBL design, as shown in Figure 4b. In the In limited growth regime the height depends on the NW pitch, as long as it is smaller than the adatom characteristic diffusion length. We found that using an oxide mask between the Au seed particles makes the heights of the kinks independent of the NW pitch down to at least 1.5 micrometer. We propose that the role of the In adatom surface diffusion is significantly reduced due to roughness of the InAs planar growth on the oxide mask. Since the oxide is overgrown in the early stages of growth, we also do not expect that the growth rate is affected by NW pitch dependent secondary reabsorption of group III atoms, which was previously described by Dalacu et. al. ${ }^{39}$. Therefore, we achieve stable growth rates even for seed patterns with wide local spread in the NW pitch. In Figure 4b, we measured the kink height of $50 \mathrm{NWs}$ for three different volumes of Au seed particles and six different NW pitches. Each measurement was fitted with a normal distribution, yielding the mean NW kink height and its standard deviation. The total average of the standard deviations of the NW kink height $(120 \pm 23$ $\mathrm{nm}$ ) was found to be comparable to the NW diameter, even though the NW pitch varies within each data point. This allows for high yield growth of complex NW networks, by merging multiple kinked NWs in the same plane and independent of in-plane network geometry. In Figure 4c we show an example of how hexagonal substrate seed patterning can be used to synthesize larger NW networks. Moreover, individual single crystalline structures, such as loops, double crosses, etc., based on the kinked-planar NC synthesis can be grown from specifically designed patterns (Figure 4d). However, the six-fold symmetry of the planar NW growth intrinsically limits the yield of specific types of structures. Integration of such NW networks, 
designed for larger electronic circuits, may well be possible with advanced transfer and/or fabrication methods ${ }^{45,46,47}$. Controlled kinking of the NW growth direction also offers various schemes for in-situ shadow masked junctions in NW heterostructures. This method is possible in MBE where the deposited materials originate from directional beam fluxes. By using the 'shadows' from other NWs that are in front of the beam, the junctions can be formed in-situ without breaking the vacuum. This opens for a whole new palet of material combinations that before were limited by the availablilty of selective etchants. A similar scheme is recently reported for NWs grown on (100) substrates by Gazibegovic et $\mathrm{al}^{17}$. If the deposited materials are non-diffusive this method produces well defined junctions in the shell of core-shell NW heterostructures, while preventing any undesirable damage to the NW surface. Figure 4e shows a well-defined shadow in two facet $\mathrm{Al}$ shell of a kinked InAs/Al NW heterostructure. The potential of kinked NW shadowing is extended, when the NWs are deterministically kinked in different heights by controlling the volume of the seed particle, see Figure $4 \mathrm{f}$. In this case, a thin Al film is deposited on the top facet of a highlighted kinked NW and shadowed by neighbouring NWs. 

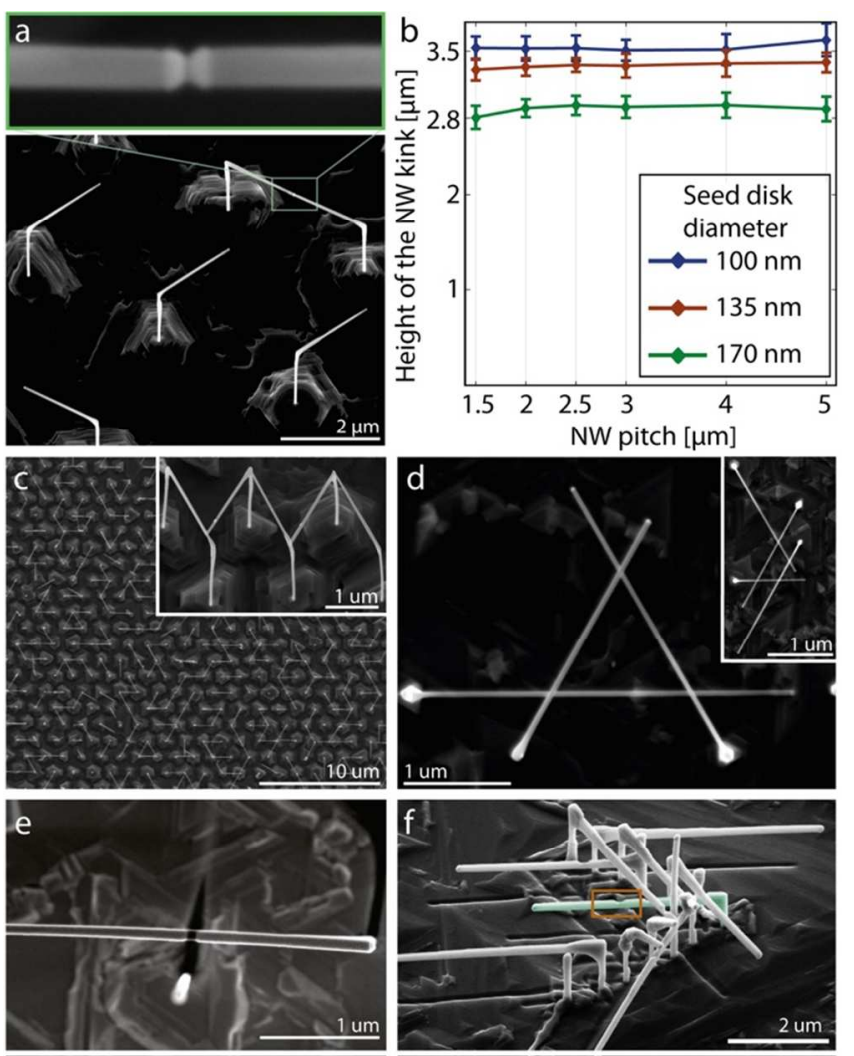

Figure 4. Scaling planar NW networks and in-situ shadow mask NWs a) Tilted SEM image of kinked NW array, with a zoom-in on two horizontally aligned kinked nanowires. b) Average NW kink height measured as a function of the NW pitch for three different sizes of EBL defined $10 \mathrm{~nm}$ thick Au disk diameter (50 NWs per data point). c) SEM image of an ensemble of more complex nanowire networks grown from hexagonal seed particle pattern, with a tilted SEM image of a network connected from 6 NWs as an inset. d) Top-view SEM image of kinked nanowire loops, based on the kinked-planar NC synthesis approach. e) Top-view SEM image of a kinked InAs/Al NW heterostructure, where the Al deposition is in-situ selectively shadowed by [0001]B NW. f) Side-view SEM image of kinked InAs/Al NW array, where control over NW kink height is used to form shadows in the one facet Al layer in-situ.

The NCs were first selected with SEM and then transferred with manipulator needles to prepatterned $\mathrm{Si} / \mathrm{SiO}_{2}$ substrates for device fabrication (S2) and room-temperature 4-probe resistivity characterization, see Figure 5. In various measurements of different WZ/ZB/WZ NCs, we did not observe a noteworthy dependence of the resistivity on the distance from the junction to the voltage probes. Other important points are that for these measurements the extracted resistivities are on average one order of magnitude higher than typical values for single NWs and 
surprisingly do not show a dependence on the path through the junction. In Figure 5a we show measurements of a double $\mathrm{WZ} / \mathrm{ZB} / \mathrm{WZ} \mathrm{NC}$ device, which was characterized in order to quantify resistance contributions of both junctions. Setting up sufficient combinations of measurements, we can extract resistances of the different junctions $R_{1}, R_{2}$ and the resistivity of the bare WZ NW segments (see supplementary S6). The average values were extracted from 4-probe resistance measurements of eight different double cross segments, by solving four sets of three equations for variables $R_{1}, R_{2}$ and $\rho_{N W \text { calc }}$ (where $\rho_{N W \text { calc }}$ is considered to be constant for each WZ segment). The calculated resistivity of the bare NW $\left((54 \pm 2) \cdot 10^{-4} \Omega \cdot \mathrm{cm}\right)$ was found to be one order of magnitude lower than the total measured average resistivity $\left((700 \pm 75) \cdot 10^{-4} \Omega \cdot \mathrm{cm}\right)$, which includes the calculated junction resistance contributions $(5.9 \pm 0.8 \mathrm{k} \Omega$ for $\mathrm{R} 1$ and $6.3 \pm 0.9$ $\mathrm{k} \Omega$ for $\mathrm{R} 2$ ). Transport through the $\mathrm{WZ} / \mathrm{ZB} / \mathrm{WZ}$ structures is therefore highly affected by the junction region.

The measured resistance through the $\mathrm{WZ} / \mathrm{ZB} / \mathrm{WZ}$ crosses does not show a dependence on the path, i.e. there is no apparent difference in resistance whether there is a possible transport path through the incoherent WZ/WZ interface or only through the ZB segment (see Figure $3 \mathrm{~d}$ for a typical junction structure). This suggests that the resistance stems from a transport barrier that extends throughout the junction. Since the WZ/WZ interfaces are single plane and because we do not see any associated strain effects in TEM, we speculate that the resistance stems from a barrier related with band offset between the $\mathrm{ZB}$ and $\mathrm{WZ}$ phases. This is consistent with recent studies on ZB-WZ heterostructures in single InAs NWs, where the band offset is sufficient to form transport barriers and electron wells ${ }^{48,49,50}$.

For relevance in quantum transport, we further speculate that the presence of a transport barrier in the junction may limit phase coherent transport between the WZ terminals. On the other hand, 
the ZB segment may form a well-defined quantum well, and as well-defined coherent polytypism in NWs have previously shown promise in quantum dot experiments and could serve as an interesting geometry with multi-terminal contacts to quantum wells.

Transport through the kinked-vertical NCs also depends on the branch combination (Figure $5 b)$, but is in this case solely related to compositional changes. The measured average resistivity through the NC $\left((10 \pm 9) \cdot 10^{-4} \Omega \cdot \mathrm{cm}\right)$ increases by one order of magnitude $\left((101 \pm 8) \cdot 10^{-4} \Omega \cdot \mathrm{cm}\right)$ when branch $\mathrm{C}$ is included in the transport. We explain this by the presence of a $\mathrm{Ga}$ rich $\mathrm{In}_{1 \text { - }}$ ${ }_{\mathrm{x}} \mathrm{Ga}_{\mathrm{x}}$ As barrier in the $<0001>\mathrm{B}$ NW, that is incorporated during the kinking growth step (see EDX analysis of the GaAs barriers in S7). Apart from that, the junction appears to be barrier free when branch $\mathrm{C}$ is excluded from the transport path. In order to avoid GaAs barrier formation, it is of interest to further develop other NW kinking mechanisms, e.g. using a temperature gradient to kink the NWs ${ }^{51,52}$.

The results of transport measurements of the kinked-planar NCs consistently show no sign of resistance associated with the junction region, as long as the two Ga contaminated segments at the kink sites are excluded from the measurements by device design, as shown in Figure 5c and supplementary S2. The measured resistance is independent of the transport path through the junction with respect to all NC segments. The averaged measured resistivity $\left((14 \pm 10) \cdot 10^{-4}\right.$ $\Omega \cdot \mathrm{cm})$ is also comparable to that of a reference kinked NW $\left(23 \cdot 10^{-4} \Omega \cdot \mathrm{cm}\right)$ shown in the inset. We expect the NC to maintain similar electronic properties in all four NC branches, which is supported by the TEM characterization suggesting that the junctions are free of structural and compositional barriers. The barrier-free transport combined with pure crystallinity makes this structure an excellent candidate for phase coherent transport in multi-terminal device geometries. We have shown that all three types of structures are feasible for electronic device fabrication, but 

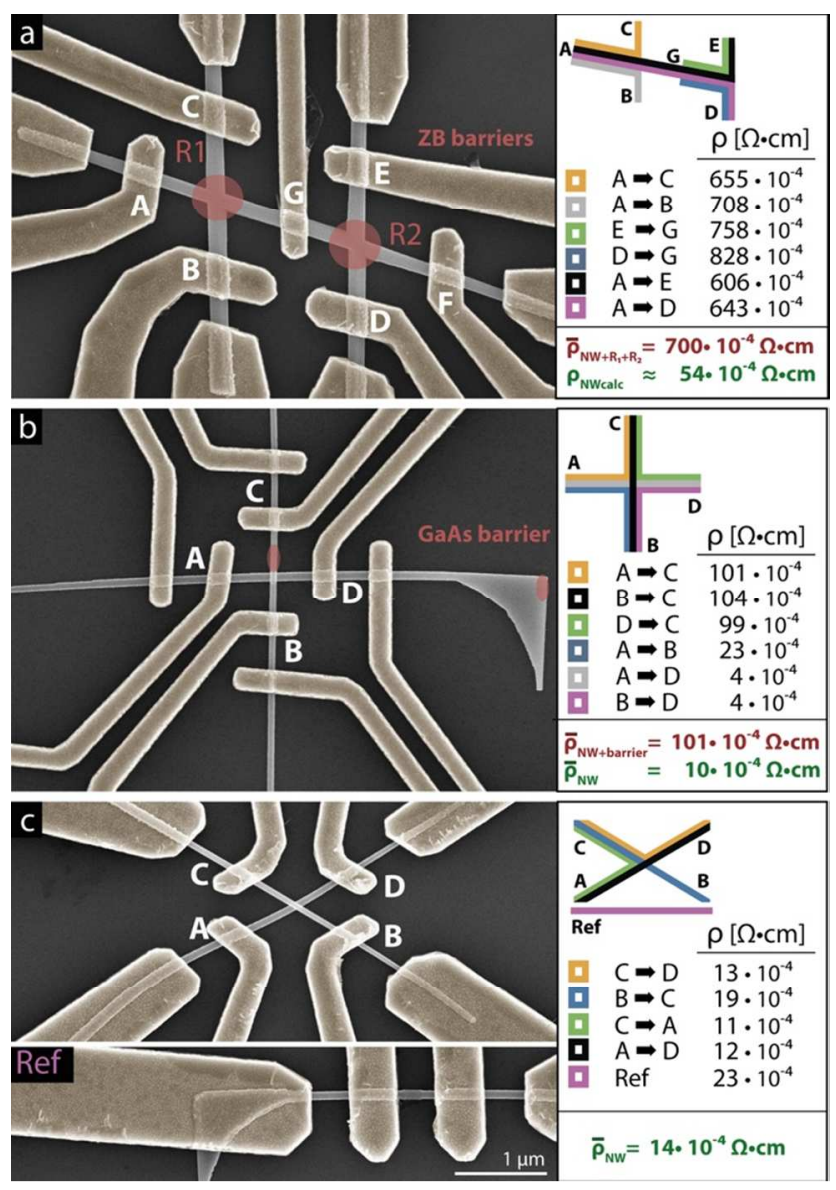

Figure 5. 4-probe characterization of InAs NCs a) Measured 4-probe resistivity through 6 different WZ/ZB/WZ double NC device segments (colored SEM image), including ZB barriers $\mathrm{R} 1$ and R2, together with averaged (from measurement) and calculated (excluding ZB barrier contribution) resistivities. b) Measured 4-probe resistivity through six different kinked-vertical NC device segments (colored SEM image) together with averaged resistivities for segments through and around GaAs barrier. c) Measured 4-probe resistivity through four different kinkedplanar NC device segments and reference nanowire (colored SEM image) and averaged resistivity of the nanowire. 
In summary, we have demonstrated methods for controlled synthesis of InAs nanocrosses. Control of the growth conditions, and rigorous substrate patterning, allow for the formation of two distinct types of single crystalline WZ NCs, 'kinked-vertical' and 'kinked-planar', in the same two-step growth process. The single crystallinity is obtained in both types of structures by using WZ [0001]B NW arrays as hexagonal basis. We show that the resistivity in both types of NCs is independent of the path through the junction. Moreover, as the kinked-planar NCs are grown parallel to the substrate, this method has potential for synthesis of epitaxially interconnected NW networks. The high electrical transparency in combination with single crystallinity of the junctions, make them excellent candidates for low temperature, phase coherent, quantum transport experiments in future studies. A third type of WZ NCs was grown directly from a cubic basis, which results in reproducible junctions with well defined inclusions of ZB. While the WZ/ZB/WZ junctions could limit phase coherence, the well-defined ZB segment could be used for multi-terminal transport experiment through a crystal phase defined quantum well.

\section{ASSOCIATED CONTENT \\ Supporting Information.}

S1: Details of growth substrate fabrication procedures. S2: Description of controlled NC mechanical transfer. S3: Close up SEM images of the junction of kinked-vertical NCs. S4: Detailed transmission electron microscope images of the kinked nanowires, with focus on defects. S5: HR TEM image of the incoherent WZ-WZ boundary in a WZ/ZB/WZ NC. S6: Description of extraction of ZB segment resistance from 4-probe measurements on [0001]X[0001] NC. S7: EDX analysis of GaAs barrier in kinked-vertical NCs. 


\author{
AUTHOR INFORMATION \\ Corresponding Author \\ *E-mail: krogstrup@,nbi.ku.dk \\ ORCID \\ P. Krogstrup 0000-0002-1930-8553
}

\title{
Author Contributions
}

The manuscript was written with contributions from all authors. All authors have given approval to the final version of the manuscript.

\section{Funding Sources}

The research was supported by Microsoft Station Q, the Danish National Science Research Foundation and the Villum Foundation.

\section{Notes}

The authors declare no competing financial interests.

\section{ACKNOWLEDGMENT}

We thank H. Shtrikman, J. Kang and H. Beidenkopf for discussions regarding growth of NWs on (100) substrates. We also thank S. Upadhyay and C. B. Sørensen for technical support. 
Modelling of the junction crystal structures was performed in software Vesta ${ }^{53}$. All NCs were characterized by scanning electron microscope JEOL 7800F as-grown and transmission electron microscopes JEOL 3000F (300 kV) and CM20 Phillips LaB6 (200kv).

\begin{abstract}
ABBREVIATIONS
NW, Nanowire; NC, Nanocross; ZB, Zinc Blende; WZ, Wurtzite; MBE, molecular beam epitaxy; VLS, vapor liquid solid; SEM, Scanning electron microscopy; TEM, Transmission electron microscopy; EBL, Electron beam lithography.

REFERENCES
\end{abstract}

${ }^{1}$ Joyce, H. J.; Wong-Leung, J.; Gao, Q.; Tan, H. H.; Jagadish, C. Nano Lett. 2010, 10 (3), $908-$ 915.

${ }^{2}$ Dick, K. A.; Caroff, P.; Bolinsson, J.; Messing, M. E.; Johansson, J.; Deppert, K.; Wallenberg, L. R.; Samuelson, L. Semicond. Sci. Technol. 2010, 25 (2), 24009.

${ }^{3}$ Fortuna, S. A.; Li, X. Semicond. Sci. Technol. 2010, 25 (2), 24005.

${ }^{4}$ Lauhon, L. J.; Gudiksen, M. S.; Wang, D.; Lieber, C. M. Nature 2002, 420 (6911), 57-61.

${ }^{5}$ Krogstrup, P.; Ziino, N. L. B.; Chang, W.; Albrecht, S. M.; Madsen, M. H.; Johnson, E.; Nygård, J.; Marcus, C. M.; Jespersen, T. S. Nat. Mater. 2015, 14 (4), 400-406.

${ }^{6}$ Gooth, J.; Borg, M.; Schmid, H.; Schaller, V.; Wirths, S.; Moselund, K.; Luisier, M.; Karg, S.; Riel, H. Nano Lett. 2017, 17 (4), 2596-2602.

${ }^{7}$ Dick, K. A.; Deppert, K.; Larsson, M. W.; Mårtensson, T.; Seifert, W.; Wallenberg, L. R.; Samuelson, L. Nat. Mater. 2004, 3 (6), 380-384. 
${ }^{8}$ Wang, D.; Qian, F.; Yang, C.; Zhong, Z.; Lieber, C. M. Nano Lett. 2004, 4 (5), 871-874.

${ }^{9}$ Dick, K. A.; Deppert, K.; Karlsson, L. S.; Seifert, W.; Wallenberg, L. R.; Samuelson, L. Nano Lett. 2006, 6 (12), 2842-2847.

${ }^{10}$ Dick, K. A.; Deppert, K.; Larsson, M. W.; Seifert, W.; Wallenberg, L. R.; Samuelson, L. Nanotechnology 2007, 18 (3), 35601.

\footnotetext{
${ }^{11}$ Suyatin, D. B.; Sun, J.; Fuhrer, A.; Wallin, D.; Fröberg, L. E.; Karlsson, L. S.; Maximov, I.; Wallenberg, L. R.; Samuelson, L.; Xu, H. Q. Nano Lett. 2008, 8 (4), 1100-1104.
}
${ }^{12}$ Dalacu, D.; Kam, A.; Austing, D. G.; Poole, P. J. Nano Lett. 2013, 13 (6), 2676-2681.
${ }^{13}$ Kang, J.-H.; Cohen, Y.; Ronen, Y.; Heiblum, M.; Buczko, R.; Kacman, P.; Popovitz-Biro, R.; Shtrikman, H. Nano Lett. 2013, 13 (11), 5190-5196.
${ }^{14}$ Plissard, S. R.; van Weperen, I.; Car, D.; Verheijen, M. A.; Immink, G. W. G.; Kammhuber, J.; Cornelissen, L. J.; Szombati, D. B.; Geresdi, A.; Frolov, S. M.; Kouwenhoven, L. P.; Bakkers, E. P. A. M. Nat. Nanotechnol. 2013, 8 (11), 859-864.

\footnotetext{
${ }^{15}$ Car, D.; Wang, J.; Verheijen, M. A.; Bakkers, E. P. A. M.; Plissard, S. R. Adv. Mater. 2014, $26(28), 4875-4879$.

${ }^{16}$ Rieger, T.; Rosenbach, D.; Vakulov, D.; Heedt, S.; Schäpers, T.; Grützmacher, D.; Lepsa, M. I. Nano Lett. 2016, 16 (3), 1933-1941.
} 
${ }^{17}$ Gazibegovic, S.; Car, D.; Zhang, H.; Balk, S. C.; Logan, J. A.; de Moor, M. W. A.; Cassidy, M. C.; Schmits, R.; Xu, D.; Wang, G.; Krogstrup, P.; Veld, R. L. M. O. het; Shen, J.; Bouman, D.; Shojaei, B.; Pennachio, D.; Lee, J. S.; van Veldhoven, P. J.; Koelling, S.; Verheijen, M. A.; Kouwenhoven, L. P.; Palmstrøm, C. J.; Bakkers, E. P. A. M. arXiv:1705.01480v2 2017.

${ }^{18}$ Aasen, D.; Hell, M.; Mishmash, R. V.; Higginbotham, A.; Danon, J.; Leijnse, M.; Jespersen, T. S.; Folk, J. A.; Marcus, C. M.; Flensberg, K.; Alicea, J. Phys. Rev. X 2016, 6 (3), 31016.

${ }^{19}$ Kang, J.-H.; Galicka, M.; Kacman, P.; Shtrikman, H. Nano Lett. 2017, 17 (1), 531-537.

${ }^{20}$ Tanta, R.; Kanne, T.; Amaduzzi, F.; Liao, Z.; Madsen, M. H.; Alarcón-Lladó, E.; Krogstrup, P.; Johnson, E.; Morral, A. F. i; Vosch, T.; Nygård, J.; Jespersen, T. S. Nanotechnology 2016, 27 (30), 305704.

${ }^{21}$ Potts, H.; Morgan, N. P.; Tütüncüoglu, G.; Friedl, M.; Morral, A. F. i. Nanotechnology 2017, $28(5), 54001$.

${ }^{22}$ Krogstrup, P.; Yamasaki, J.; Sørensen, C. B.; Johnson, E.; Wagner, J. B.; Pennington, R.; Aagesen, M.; Tanaka, N.; Nygård, J. Nano Lett. 2009, 9 (11), 3689-3693.

${ }^{23}$ Musin, I. R.; Filler, M. A. Nano Lett. 2012, 12 (7), 3363-3368.

${ }^{24}$ Hillerich, K.; Dick, K. A.; Wen, C.-Y.; Reuter, M. C.; Kodambaka, S.; Ross, F. M. Nano Lett. 2013, 13 (3), 903-908.

${ }^{25}$ Tian, B.; Xie, P.; Kempa, T. J.; Bell, D. C.; Lieber, C. M. Nat. Nanotechnol. 2009, 4 (12), 824-829. 


\footnotetext{
${ }^{26}$ Yuan, X.; Caroff, P.; Wong-Leung, J.; Fu, L.; Tan, H. H.; Jagadish, C. Adv. Mater. 2015, 27 (40), 6096-6103.

${ }^{27}$ Madras, P.; Dailey, E.; Drucker, J. Nano Lett. 2009, 9 (11), 3826-3830.

${ }^{28}$ Zhang, M.; Wang, F.; Wang, C.; Wang, Y.; Yip, S.; Ho, J. C. Nanoscale Res. Lett. 2014, 9 (1), 211.
}

${ }^{29}$ Lenrick, F.; Ek, M.; Deppert, K.; Samuelson, L.; Reine Wallenberg, L. Nano Res. 2014, 7 (8), 1188-1194.

${ }^{30}$ Zannier, V.; Ercolani, D.; Gomes, U. P.; David, J.; Gemmi, M.; Dubrovskii, V. G.; Sorba, L. Nano Lett. 2016, 16 (11), 7183-7190.

${ }^{31}$ Hocevar, M.; Immink, G.; Verheijen, M.; Akopian, N.; Zwiller, V.; Kouwenhoven, L.; Bakkers, E. Nat. Commun. 2012, 3, 1266.

${ }^{32}$ Yan, X.; Zhang, X.; Li, J.; Wu, Y.; Ren, X. Appl. Phys. Lett. 2015, 107 (2), 23101.

${ }^{33}$ Lukyanov, A. V.; Likhtman, A. E. ACS Nano 2016, 10 (6), 6045-6053.

${ }^{34}$ Krogstrup, P.; Jørgensen, H. I.; Johnson, E.; Madsen, M. H.; Sørensen, C. B.; Morral, A. F. i; Aagesen, M.; Nygård, J.; Glas, F. J. Phys. D. Appl. Phys. 2013, 46 (31), 313001.

${ }^{35}$ Dai, X.; Dayeh, S. A.; Veeramuthu, V.; Larrue, A.; Wang, J.; Su, H.; Soci, C. Nano Lett. 2011, 11 (11), 4947-4952.

\footnotetext{
${ }^{36}$ Li, Z.-A.; Möller, C.; Migunov, V.; Spasova, M.; Farle, M.; Lysov, A.; Gutsche, C.; Regolin, I.; Prost, W.; Tegude, F.-J.; Ercius, P. J. Appl. Phys. 2011, 109 (11), 114320.

37 Wang, J.; Plissard, S.; Hocevar, M.; Vu, T. T. T.; Zehender, T.; Immink, G. G. W.; Verheijen, M. A.; Haverkort, J.; Bakkers, E. P. A. M. Appl. Phys. Lett. 2012, 100 (5), 53107.

${ }^{38}$ Aagesen, M.; Johnson, E.; Sørensen, C. B.; Mariager, S. O.; Feidenhans’1, R.; Spiecker, E.; Nygård, J.; Lindelof, P. E. Nat. Nanotechnol. 2007, 2 (12), 761-764.
} 
${ }^{39}$ Dalacu, D.; Kam, A.; Guy Austing, D.; Wu, X.; Lapointe, J.; Aers, G. C.; Poole, P. J Nanotechnology 2009, 20 (39), 395602.

40 Kelrich, A.; Calahorra, Y.; Greenberg, Y.; Gavrilov, A.; Cohen, S.; Ritter, D. Nanotechnology 2013, 24 (47), 475302.

${ }^{41}$ Ghosh, S. C.; Kruse, P.; LaPierre, R. R.; Nanotechnology 2009, 20 (11), 115602.

${ }^{42}$ Whiticar, A. M.; Mårtensson, E. K.; Nygård, J.; Dick, K. A.; Bolinsson, J. Nanotechnology 2017, 28 (20), 205702.

${ }^{43}$ Seth A Fortuna, X. L. Semicond. Sci. Technol. Semicond. Sci. Technol 2010, 25 (2), 2400524016.

${ }^{44}$ Fonseka, H. A.; Caroff, P.; Wong-Leung, J.; Ameruddin, A. S.; Tan, H. H.; Jagadish, C. ACS Nano 2014, 8 (7), 6945-6954.

${ }^{45}$ Freer, E. M.; Grachev, O.; Duan, X.; Martin, S.; Stumbo, D. P. Nat. Nanotechnol. 2010, 5 (8), 625-625.

${ }^{46}$ Standing, A. J.; Assali, S.; Haverkort, J. E. M.; Bakkers, E. P. A. M. Nanotechnology 2012, $23(49), 495305$.

47 Meyer-Holdt, J.; Kanne, T.; Sestoft, J. E.; Gejl, A.; Zeng, L.; Johnson, E.; Olsson, E.; Nygård, J.; Krogstrup, P. Nanotechnology 2016, 27 (36), 365603.

${ }^{48}$ Chen, I.-J.; Lehmann, S.; Nilsson, M.; Kivisaari, P.; Linke, H.; Dick, K. A.; Thelander, C. Nano Lett. 2017, 17 (2), 902-908.

${ }^{49}$ Dick, K. A.; Thelander, C.; Samuelson, L.; Caroff, P. Nano Lett. 2010, 10 (9), 3494-3499. 


\footnotetext{
${ }^{50}$ Nilsson, M.; Namazi, L.; Lehmann, S.; Leijnse, M.; Dick, K. A.; Thelander, C. Phys. Rev. B 2016, 93 (19), 195422.

${ }^{51}$ Kelrich, A.; Sorias, O.; Calahorra, Y.; Kauffmann, Y.; Gladstone, R.; Cohen, S.; Orenstein, M.; Ritter, D. Nano Lett. 2016, 16 (4), 2837-2844.

${ }^{52}$ Panciera, F.; Norton, M. M.; Alam, S. B.; Hofmann, S.; Mølhave, K.; Ross, F. M.; Nat. Commun. 2016, 7, 12271.

${ }^{53}$ Momma, K.; Izumi, F. J. Appl. Crystallogr. 2011, 44 (6), 1272-1276.
} 

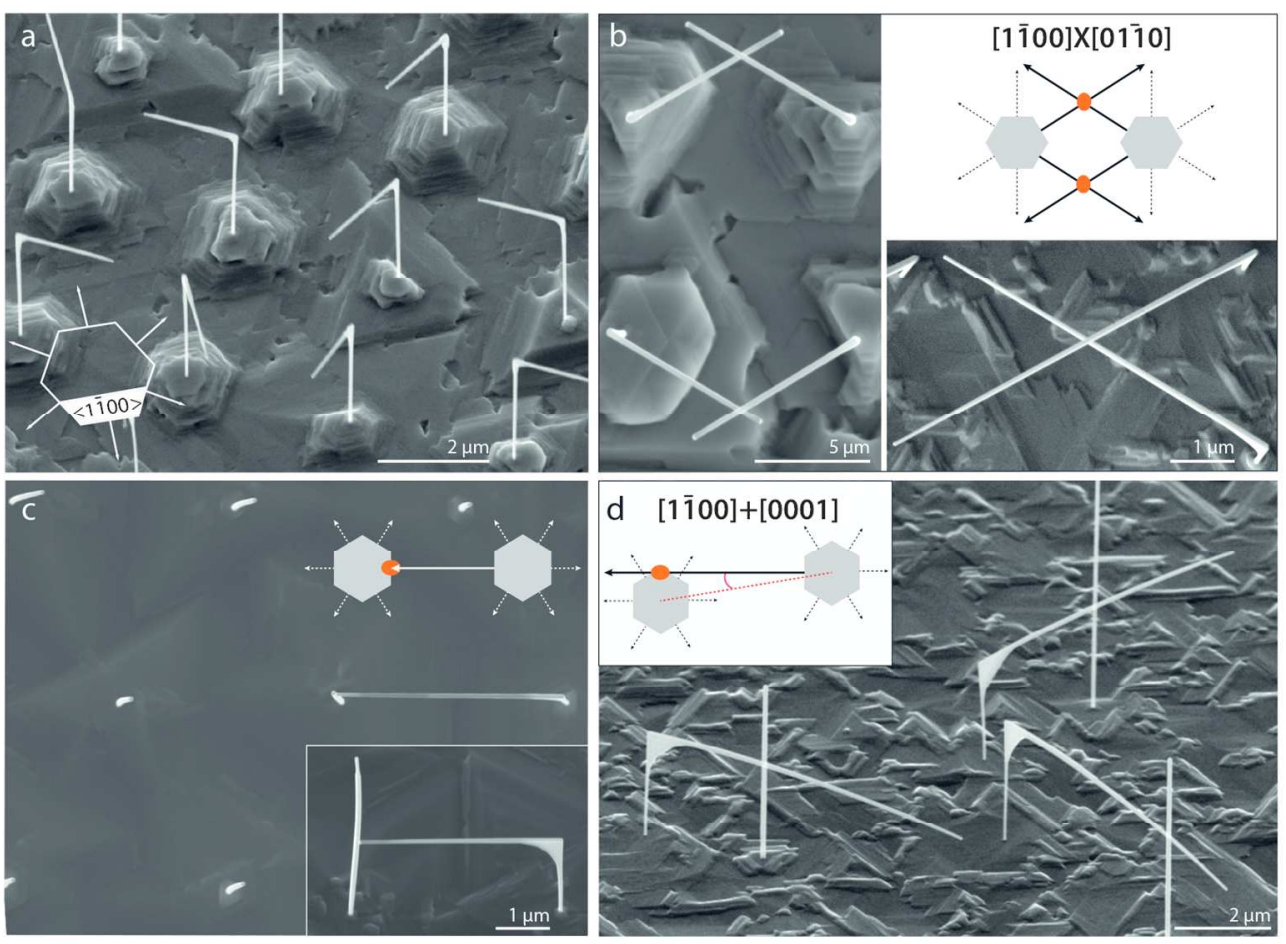

$139 \times 101 \mathrm{~mm}(300 \times 300$ DPI $)$ 

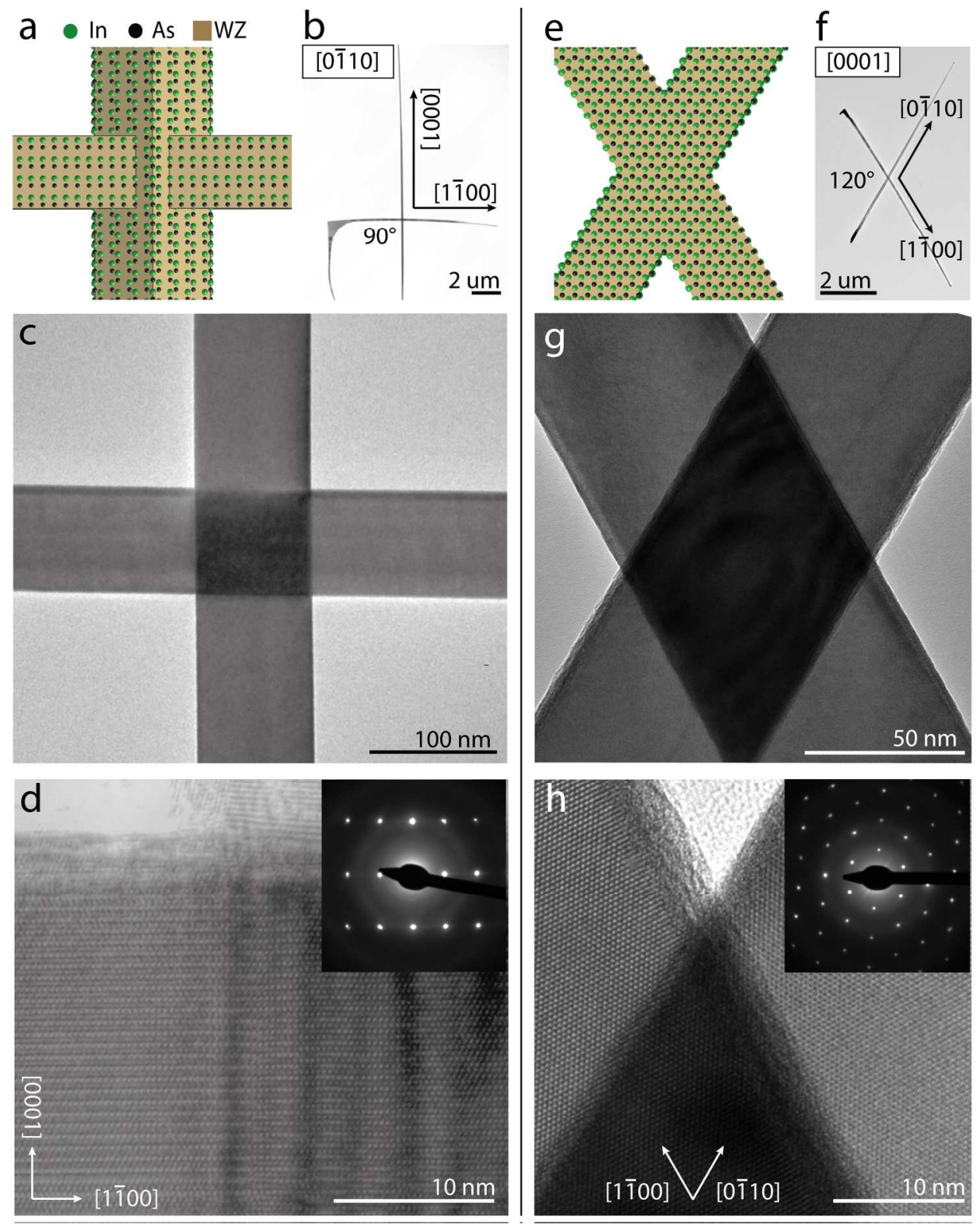

$722 \times 884 \mathrm{~mm}(72 \times 72$ DPI $)$ 

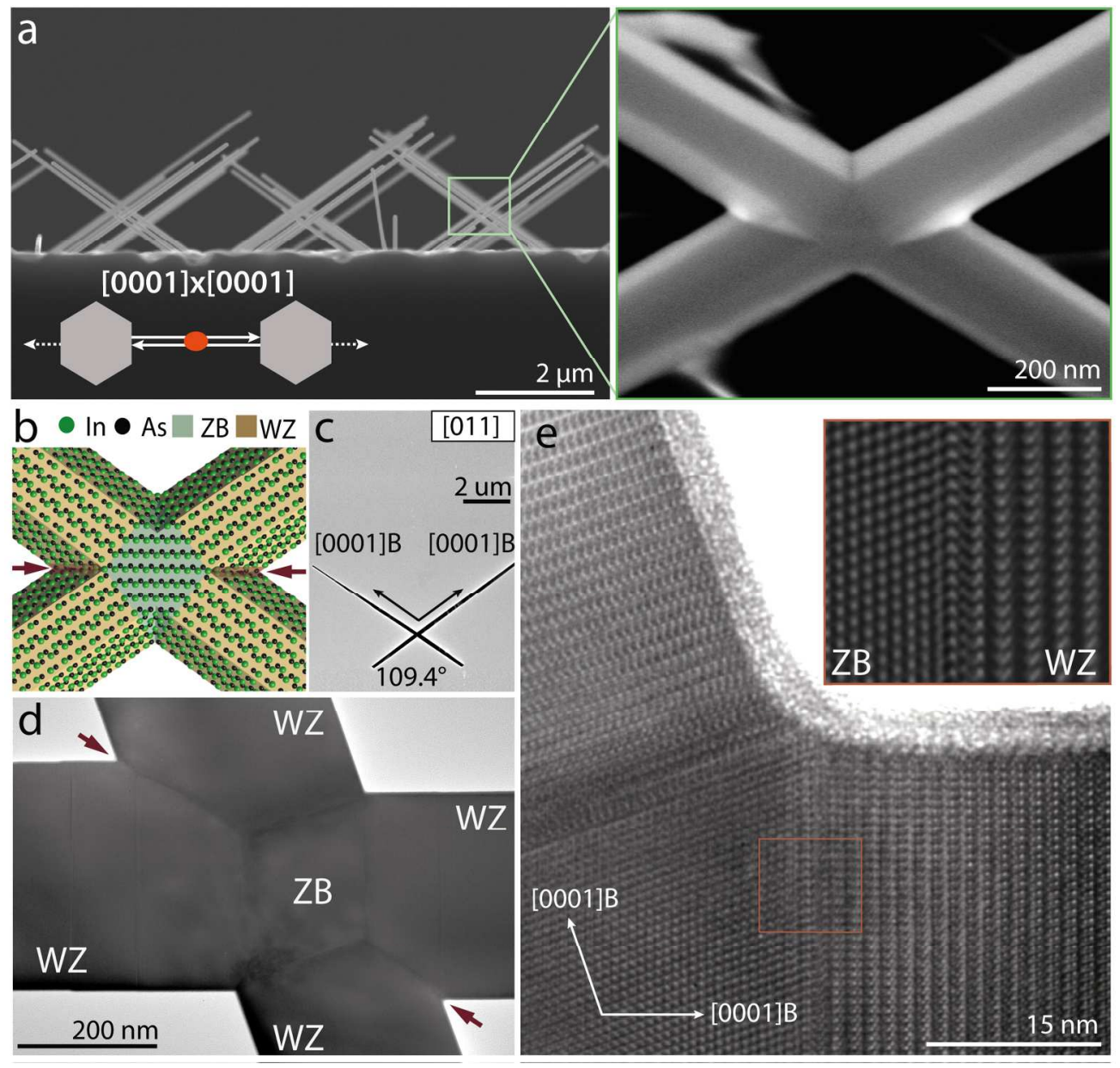

$722 \times 683 \mathrm{~mm}(72 \times 72 \mathrm{DPI})$ 

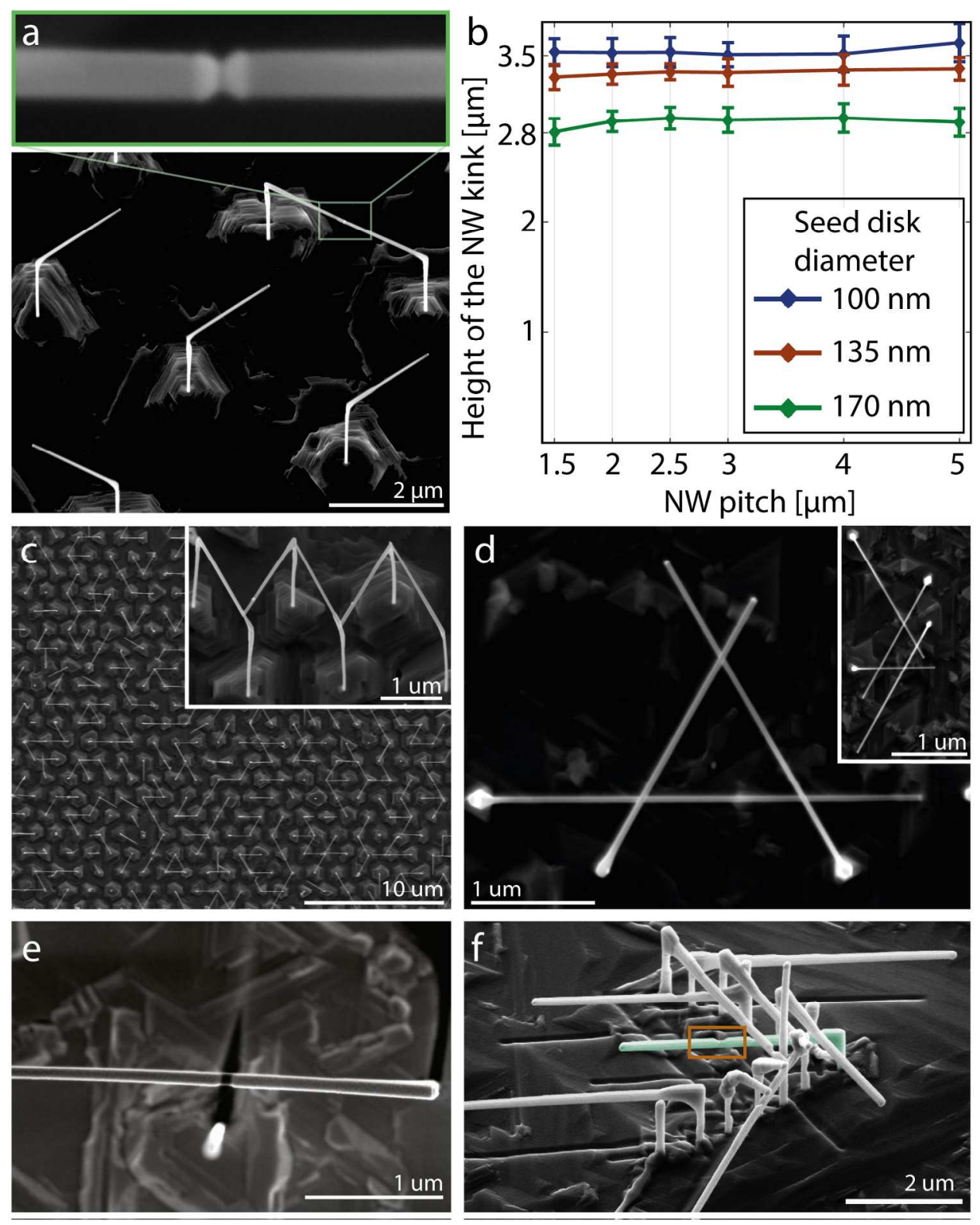

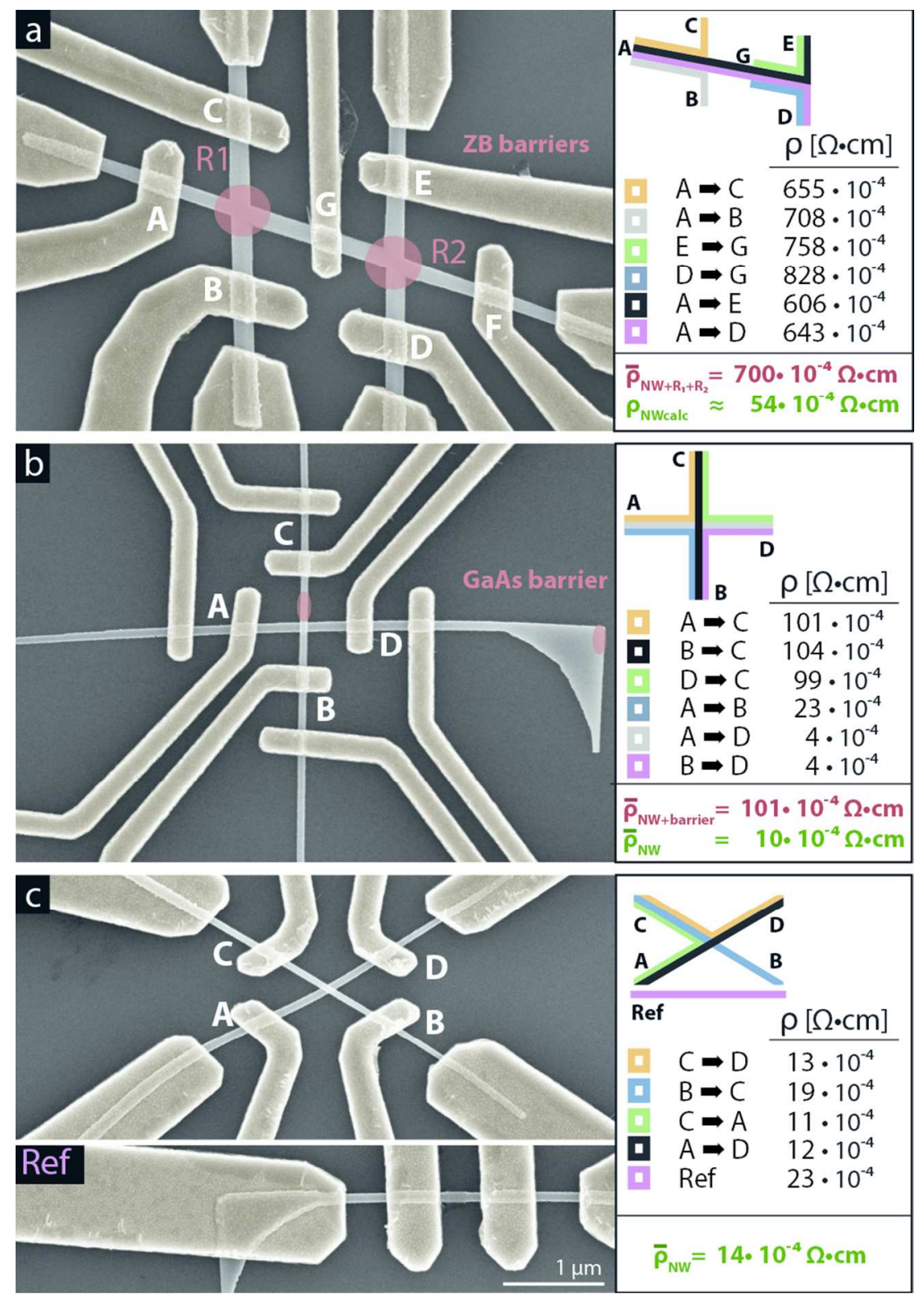

$85 \times 121 \mathrm{~mm}(300 \times 300$ DPI $)$ 


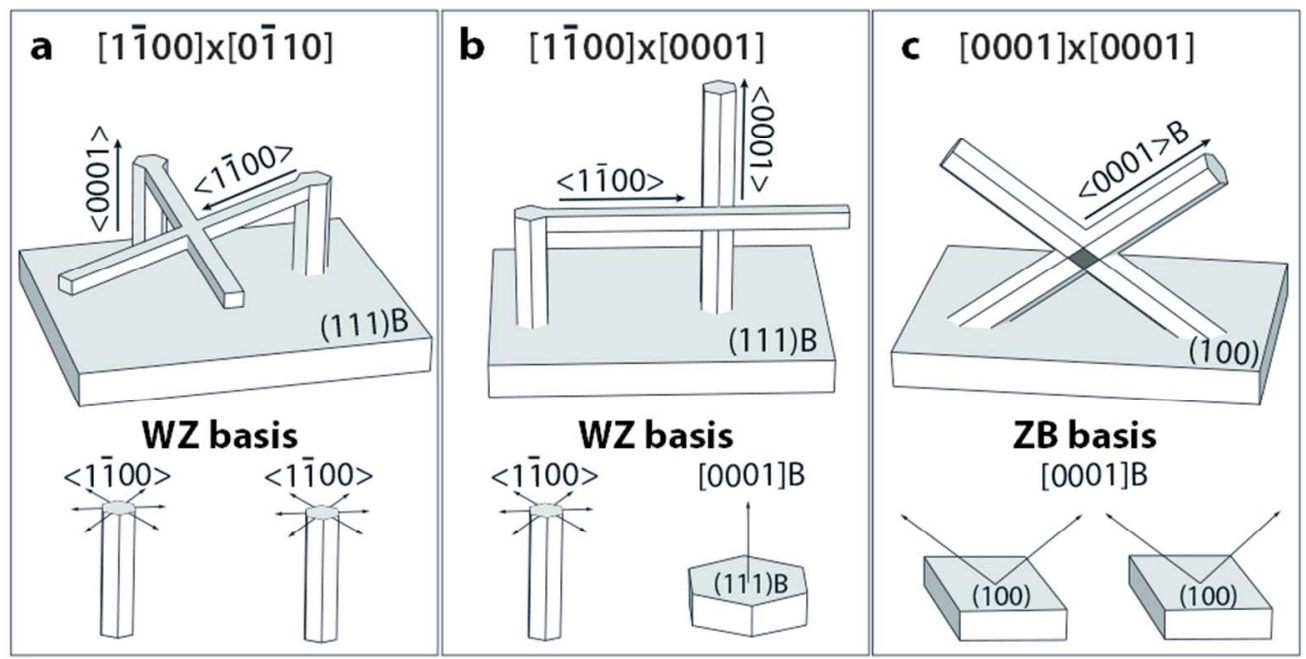

$80 \times 40 \mathrm{~mm}(300 \times 300$ DPI $)$ 